\title{
Fuerza innovadora
}

\section{Innovative power}

El año 2019 la Facultad de Estomatología de la Universidad Peruana Cayetano Heredia cumplirá cincuenta años de existencia. Nació al Perú y al mundo con el ímpetu de innovar la educación odontológica preparando nuevas generaciones de odontólogos formados en las humanidades, las ciencias, las artes y en el afán de servir a su pueblo. Desde su fundación se declaró como una institución experimental, que se proponía colocar al Perú en una posición digna en el concierto de la educación odontológica de América Latina.

No fue fácil lograr que un grupo de dentistas sanmarquinos se hicieran de un lugar en una universidad médica. Luego de largos meses de planeación, la nueva facultad fue creada en sesión de Consejo Universitario presidido por el Dr. Alberto Hurtado Abadía, al apuntar la noche del once de diciembre de 1969 en la sala de sesiones del Consejo, en el local central de la Universidad, en el distrito de San Martín de Porres de la ciudad de Lima, Perú.

Con el apoyo de la Organización Panamericana de la Salud para asesoría técnica y de la Fundación W.K.Kellogg para el financiamiento, se inició el proceso de formación y desarrollo, cuya primera etapa fue el reclutamiento y preparación académica a nivel superior, de jóvenes odontólogos procedentes de las facultades de odontología de las universidades de San Marcos y San Luis Gonzaga, para ocupar cargos en la docencia.

La segunda tarea fue la construcción de un currículo odontológico basado en el estudio de las necesidades de salud bucal y la determinación de las funciones, que el graduado debería poder ejercer al término de sus estudios, para atender a tales necesidades. Fue decisión del grupo de fundadores de la Facultad no adoptar ni adaptar currículos existentes en otras instituciones del Perú o de otros países. Los fundadores y los jóvenes profesores incorporados, dedicaron sesiones diarias durante un año para construir un currículo original. Por sus características y propósitos, el currículo resultante exigió una nueva metodología para su ejecución.

Junto con el currículo por competencias profesionales, se impartían los cursos de humanidades y ciencias básicas y biológicas, que quedaron a cargo de la Facultad de Ciencias de la Universidad, constituyendo los estudios generales o ciclo básico.

Distinto a la habitual enseñanza por asignaturas el nuevo currículo adoptaba el sistema de enseñanza modular centrado en el dominio de las funciones clínicas. Cada módulo contenía los aspectos cognitivos, afectivos y psicomotores. La participación de los alumnos se materializaba mediante la preparación del contenido teórico y su discusión, y luego, en la actividad clínica con el paciente. Podemos afirmar que nuestra Institución fue pionera en la enseñanza por competencias, que entonces llamábamos funciones.

El aprendizaje pre clínico se juntó modularmente al aprendizaje clínico, dejando de lado las muchas horas que los currículos tradicionales dedican a la pre clínica. Se resolvió no utilizar maniquís, optándose por el contacto temprano con el paciente, comenzando con la atención preventiva en el paciente niño. 
El énfasis puesto en el componente social de la educación superior se concretó en el trabajo en comunidades urbanas carentes y en el internado rural, que exigía una permanencia de seis meses en comunidades distantes, trabajando con autonomía bajo supervisión indirecta.

El currículo alentaba la utilización de los métodos de la investigación para el aprendizaje. Desde el primer año de los estudios los alumnos eran estimulados para recoger información sistematizada en torno a preguntas surgidas de las discusiones grupales y de los contenidos prácticos del aprendizaje.

Los positivos resultados del nuevo currículo se constataron desde las primeras promociones. Sus integrantes demostraban en el medio laboral una excelente capacidad para el diagnóstico y tratamiento de sus pacientes. Durante los años transcurridos los exalumnos han alcanzado notables puestos y distinciones en prestigiosas instituciones nacionales y extranjeras, como son la International Association of Dental Research, el Centro para el Control de las enfermedades CDC, la American Dental Association, la universidad de Sao Paulo y la Asociación Odontopediátrica Mundial. Otros exalumnos destacan en la docencia y la investigación, ocupando cargos de dirección en universidades extranjeras. La información recibida de instituciones donde los exalumnos hicieron estudios de postgrado como el Eastman Dental Center, dan cuenta de la excelencia de su rendimiento y logros.

Con el crecimiento indiscriminado de nuevas facultades de odontología en el país se ha notado una disminución del número y de la preparación de los postulantes. Este hecho ha puesto en guardia a las autoridades y profesores. Una disminución en la preparación de los postulantes lleva necesariamente a un aumento de la calidad de la enseñanza, a un énfasis en la preparación de los profesores, así como en la mayor dedicación de estos a la docencia y a la investigación.

Transcurridos 47 años desde su creación, cabe destacar el prestigio nacional e internacional rápidamente alcanzado por la Facultad. Uno de los indicadores de ello es la matrícula de nacionales y extranjeros de distintas universidades en los cursos de especialización del postgrado, así como en los cursos para optar por los grados de maestría y doctorado en Estomatología.

La calidad de Fe-UPCH se manifiesta también en su producción científica, tanto en el material que se publica en la Revista Estomatológica Herediana como en el numeroso acervo de libros preparados por el profesorado. Títulos de dicho acervo han sido adoptados como libros de texto en facultades de odontología de distintos países de América Latina.

El continuo desarrollo de la Facultad llevó a la construcción de la unidad para la formación de técnicos y asistentes dentales y la edificación de la escuela de postgrado, ambas unidades logradas con recursos propios. Entre los legados de la nueva facultad se reconocen: el método de construcción curricular, la enseñanza modular de funciones y competencias clínicas, sistematizada mediante una original Matriz Taxonómica; el internado rural sistematizado y el internado hospitalario a dedicación exclusiva.

Quienes fundaron la Fe-UPCH y sus actuales profesores y alumnos de pre y postgrado pueden sentirse orgullosos de la institución que respalda la calidad de su formación y marca su desarrollo profesional. El prestigio de sus exalumnos constituye un auténtico reconocimiento a la calidad del proceso formativo de la Institución.

Roberto J. Beltrán Neira ${ }^{1, a}$

Facultad de Estomatología, Universidad Peruana Cayetano Heredia. Lima, Perú.

Profesor Emerito, Promotor, Fundador y primer Decano de la Facultad de Estomatología. 